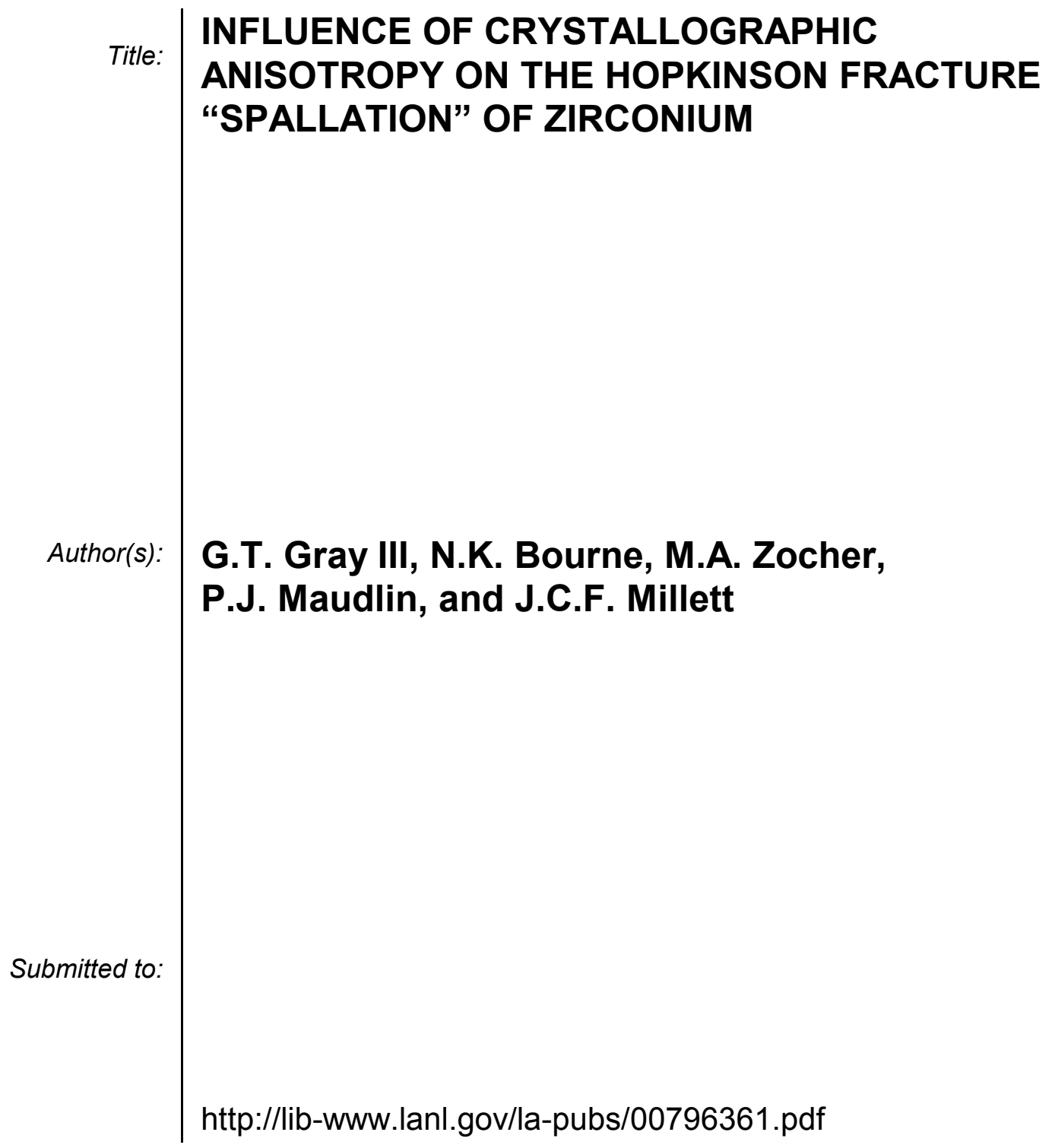

Los Alamos National Laboratory, an affirmative action/equal opportunity employer, is operated by the University of California for the U.S. Department of Energy under contract W-7405-ENG-36. By acceptance of this article, the publisher recognizes that the U.S. Government retains a nonexclusive, royaltyfree license to publish or reproduce the published form of this contribution, or to allow others to do so, for U.S. Government purposes. Los Alamos National Laboratory requests that the publisher identify this article as work performed under the auspices of the U.S. Department of Energy. Los Alamos National Laboratory strongly supports academic freedom and a researcher's right to publish; as an institution, however, the Laboratory does not endorse the viewpoint of a publication or guarantee its technical correctness. 


\title{
INFLUENCE OF CRYSTALLOGRAPHIC ANISOTROPY ON THE HOPKINSON FRACTURE “SPALLATION” OF ZIRCONIUM
}

\author{
G.T. Gray III, N.K. Bourne*, M.A. Zocher, P.J. Maudlin, and J.C.F. Millett**, \\ Los Alamos National Laboratory, Los Alamos, NM 87545 \\ * Royal Military College of Science, Shrivenham, Swindon, SN6 8LA, UK. \\ ** Cavendish Laboratory, University of Cambridge, Cambridge, CB3 OHE, UK
}

The influence of texture on the spallation (Hopkinson fracture) of low-symmetry metals (e.g. Zr, U, or Sn) has seen limited study. In this study, the Hopkinson fracture of annealed, high-purity $\mathrm{Zr}$ has been probed as a function of crystallographic texture. The quasi-static yield strength of the $\mathrm{Zr}$ studied is $\approx 2.5 \mathrm{x}$ higher in the plate's through-thickness direction compared to that measured in-plane due to a pronounced basal texture. The pullback signals of each orientation shocked to $5 \mathrm{GPa}$ were found to be insensitive to the texture, although the HEL's and the soft-recovered, incipiently-spalled samples exhibited differences in their damage evolution. The VISAR wave profiles were modeled using a 3-D finite-volume method (FVM) continuum code. The VISAR, post-spallation metallographic observations, and modeling analysis are discussed with reference to the HEL and texture.

\section{INTRODUCTION}

While the influence of crystallographic texture on metal plasticity has seen in-depth investigation over the last three decades $(1,2)$, the role of texture effects on fracture remains less extensively studied (3). Texture effects on fracture can be microscopic in nature, such as the role of crystallographic orientation on cleavage, or macroscopic due to the influence of texture-induced elastic and plastic anisotropy on crack growth in a polycrystal. The development of physically-based damage-evolution and fracture models requires a detailed understanding of the synergistic effects between both influences.

Shock-induced fracture in materials due to the interaction of the reflected wave from the rear surface of an assembly with the tail of the release from the impactor, was first discovered by Bertram Hopkinson (4). This process (previously termed "Hopkinson fracture" (5), and more generically called spallation (6)) is known to be influenced by microstructural variables in materials (6). Singlecrystal studies on Be have shown that single-crystal anisotropy produces a very pronounced effect on the Hugoniot-elastic-limit (HEL) in Be (7).
The goal of this study was to quantify the influence of texture on the shock-induced dynamic fracture of strongly-textured polycrystalline zirconium $(\mathrm{Zr})$.

\section{EXPERIMENTAL TECHNIQUES}

This investigation utilized high-purity $\mathrm{Zr}$ which contained approximately $54 \mathrm{ppm} \mathrm{Hf}$ and $40 \mathrm{ppm} \mathrm{O}$ (wt. \%). This $\mathrm{Zr}$ was clock-rolled at $298 \mathrm{~K}$ then annealed at $823 \mathrm{~K}$ for 1 hour. This produced an equiaxed grain structure with a mean size of $25 \mu \mathrm{m}$ and possessing a strong basal texture with hexagonal symmetry in the plate. Peak flow stresses in quasistatic compression tests at $298 \mathrm{~K}$ due to this strong texture are approximately 2.5 times greater in the through-thickness plate direction (TT) compared to samples tested in the plane (IP) of the plate for this $\mathrm{Zr}(8)$. When tested at $76 \mathrm{~K}$, yield stresses of the TT samples were 3 times greater than the IP samples (8).

To examine the influence of loading orientation, and thereby texture, on the Hopkinson fracture response of this strongly basal textured Zr-plate, 10 
mm diameter samples were sectioned from both the TT and IP directions. Plate impact experiments were carried out using a $50 \mathrm{~mm}$ bore gas gun. $\mathrm{Zr}$ samples were machined from the TT and IP orientations and interference fitted into the center of a $50-\mathrm{mm}$ diameter "donut" of lower-purity $\mathrm{Zr}$ material possessing a higher yield strength and somewhat weaker basal texture. The differences in the textures and yield strengths between the high-purity $\mathrm{Zr}$ and the lower purity $\mathrm{Zr}$ momentum ring surrounding the sample have been detailed previously (8).

In all cases, the impactors were half-hard copper of thickness $1.12 \mathrm{~mm}$ and the $\mathrm{Zr}$ targets of thickness $2.0 \mathrm{~mm}$ so that a central spall plane was introduced. The samples were impacted at $330 \mathrm{~m} \mathrm{~s}^{-1}$ for a nominal shock pressure of $5 \mathrm{GPa}$. The impact velocity was measured to an accuracy of $0.5 \%$ using a sequential pin-shorting method and tilt was fixed to be less than $1 \mathrm{mrad}$ by means of an adjustable specimen mount. The rear surface of the target was monitored using VISAR (9) with an adapted system usingpolymer fibers to illuminate and receive phase information from the target. This data was unwrapped using the method of Hemsing (9) using all four The spalled $\mathrm{Zr}$ samples were "soft-recovered" by decelerating the shocked samples in layers of rags. Specimens for optical metallography were sectioned from the shock-recovered samples and prepared for optical metallography as previously described (8).

\section{RESULTS AND DISCUSSION}

\section{VISAR Data}

Preferred texture in polycrystals can result in orientation-dependent constitutive properties (10) and can also affect damage evolution and fracture mechanisms (3). The strong basal texture, previously shown to have a pronounced effect on the uniaxial-stress constitutive behavior at low strain rates of $\mathrm{Zr}$ (8), was found in this study to: a) significantly influence the shock "ring-up" and HEL of $\mathrm{Zr}$, while 2) the texture exhibits minimal effect on the overall spallation "pull-back" response, both in magnitude and the timing of the residual ringing (Fig. 1).

The wave profiles during shock-up are seen to display a ramped "ring-up" for the TT orientation with a break at a particle velocity of $\sim 0.08$ while the IP sample displays a more conventional jump in particle velocity at a value of $\sim 0.04$ (noted as point A) in Fig. 1. The wave profile signal for the IP sample is seen to display a somewhat steeper initial recompression after the pull-back compared to the TT sample; thereafter both samples exhibit essentially identical reverberations. The timing of the reverberations "ringing" in the spallation signal after the "pull-back" are consistent with the traditional trapping of momentum in the scab rear portion of the spalled sample, following the generation of a "free-surface" although no separation of either the TT or IP targets occurred.

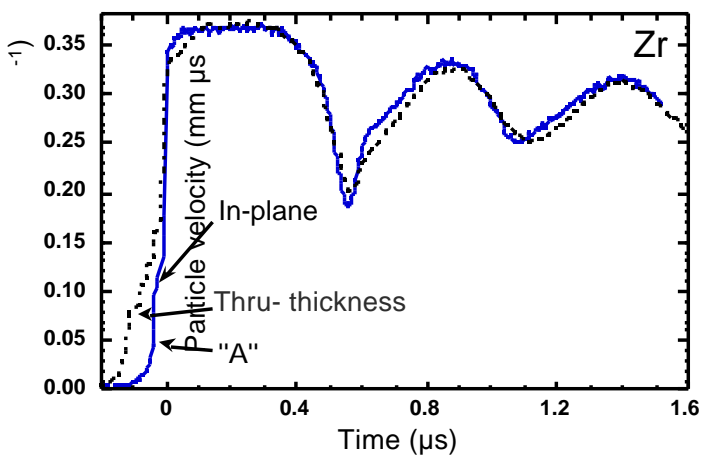

FIGURE 1. VISAR wave profiles for $\mathrm{Zr}$ spalled in the TT and IP directions.

\section{Hugoniot Elastic Limit Analysis}

The HEL for a material having both orthotropic elastic and plastic properties can be derived in laboratory frame coordinates assuming uniaxial strain (3-axis is the direction of the propagating shock) and linear elasticity:

$$
\sigma_{\mathrm{H}}=\frac{\sigma_{\mathrm{y}}}{\sqrt{\frac{\alpha_{2233}}{2}\left(\frac{\mathrm{E}_{2233}}{\mathrm{E}_{3333}}-1\right)^{2}+\frac{\alpha_{1133}}{2}\left(\frac{\mathrm{E}_{1133}}{\mathrm{E}_{3333}}-1\right)^{2}+\frac{\alpha_{1122}}{2}\left(\frac{\mathrm{E}_{1133}}{\mathrm{E}_{3333}}-\frac{\mathrm{E}_{2233}}{\mathrm{E}_{3333}}\right)^{2}}}
$$

The $E_{i j k l}$ in this equation are components of the elastic stiffness tensor, $\sigma_{\mathrm{y}}$ is the yield stress as measured in a uniaxial stress test in the TT direction (3-axis), and the $\alpha_{i j k l}$ are components of the yield surface shape tensor assuming a quadratic yield function (i.e., $f=\frac{1}{2} \alpha_{\mathrm{ijkl}} \mathrm{s}_{\mathrm{kl}} \mathrm{s}_{\mathrm{ij}}-\sigma^{2}$ ). Using properties for the high purity zirconium (2), the HEL ratio $\sigma_{\mathrm{H}, \mathrm{TT}} / \sigma_{\mathrm{H}, \mathrm{IP}}$ computed with the above $\sigma_{\mathrm{H}}$ equals 1.87 .

Numerical simulations were performed that assume the elastoplastic constitutive description given in Ref. (10), and use elastoplastic properties (2) for the zirconium sample and isotropic properties for the half-hard copper impactor. This flyer-plate problem was spatially discretized in three-dimensions for subsequent simulation using the time-dependent 
CHAD FVM code(11). An axisymmetric mesh with a total of 21,120 cells (165 TT cells, 8 radial cells and 16 azimuthal cells) was generated with the sample modeled as a two-material (specimen and ring) composite. The specimen material forms the $10 \mathrm{~mm}$ central plug pressed into an outer ring of material having potentially different elastoplastic properties due to textural and other microstructural differences(12). Stress wave reflection from the plug/ring interface due to property discontinuity could affect the wave profile results, but a radial transit time of $1.05 \mu$ s for the plug precludes any such effect during the HEL loading phase. The problem was simulated with CHAD out to $1.0 \mu \mathrm{s}$, and the results in terms of free surface particle velocity versus time are compared in Fig. 2 to the experimental data. This comparison shows good HEL agreement between data and calculations for the TT configuration (HEL at about $0.075 \mathrm{~mm} / \mu \mathrm{s}$ ), and apparent agreement for the lower amplitude IP case (HEL at about $0.035 \mathrm{~mm} / \mu \mathrm{s}$ ).

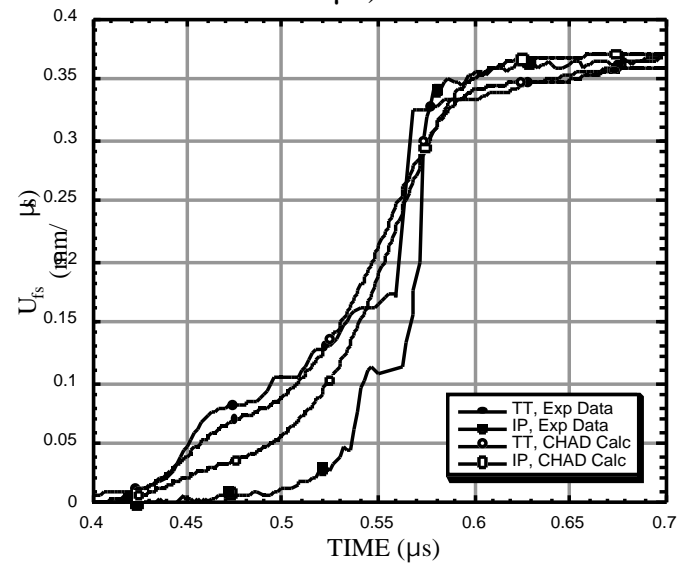

FIGURE 2. Comparison of shock profile simulation results to IP and TT test data with emphasis on the Hugoniot elastic limit.

The enhanced acceleration of the TT velocity (relative to the IP case) observed in Fig. 2 is consistent with the differing elastic moduli and stress states between the two cases. Figure 3 presents a $\pi$-plane projection of the $\mathrm{Zr}$ yield surface for the high purity clock-rolled, showing a fairly eccentric shape (a von Mises function would be round in Fig. 3). Depicted are the two stress states and plastic straining directions for the TT and IP cases. Note in this figure that the TT state is uniaxial in both stress and plastic straining, whereas, the IP state is completely $3 \mathrm{D}$ in stress and uniaxial in the plastic straining. Since the TT material direction has a faster longitudinal elastic wave speed $(4850 \mathrm{~m} / \mathrm{s}$ versus $4670 \mathrm{~m} / \mathrm{s}$ for the IP direction) and an HEL that is 1.87 larger than the IP case, acceleration in the TT direction is expected to be enhanced; material particles accelerate with a larger elastic modulus over a larger increment of elastic strain as determined by the eccentricity of the yield surface. This conclusion is supported by the data and calculations of Fig. 2.

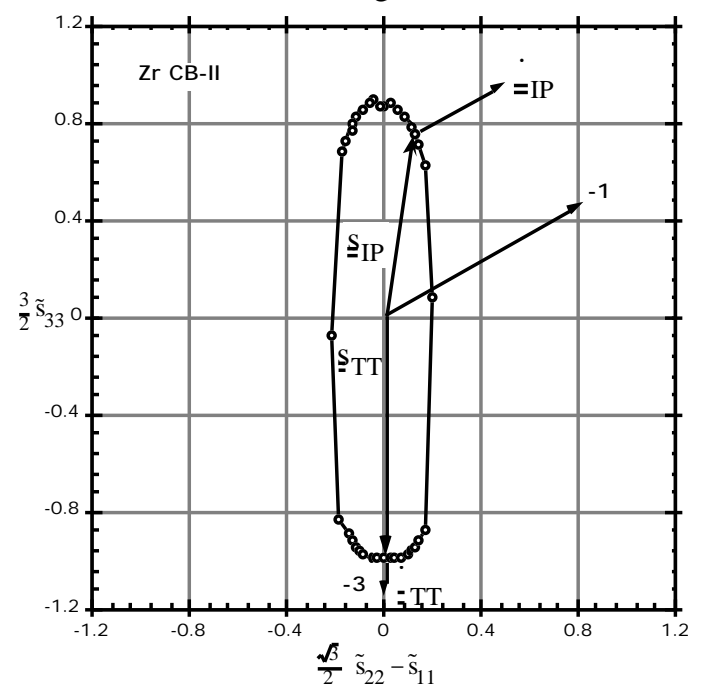

FIGURE 3: $\pi$-plane yield surface projection for the high purity clock-rolled Zr. Depicted are the TT and IP stress states and plastic straining directions.

\section{Damage Evolution}

The damage evolution in $\mathrm{Zr}$ as a function of loading orientation was evaluated through metallographic examination of the "soft-recovered" Hopkinson-spalled TT and IP samples. Figure 4 shows that the primary damage evolution in both the TT and IP samples is comprised of small $(\sim 10$ micron) nominally spherical-shaped ductile voids with no discrete separation of the spalled TT or IP samples were seen although a sizable "pull-back" signal was measured for both orientations. In addition to the ductile voids, the TT sample also displayed some ductile shear cracking, potentially contiguous although not in this figure, between voids as seen in the upper portion of the TT photomicrograph of Fig. 4.

The enhanced coalescence of the voids into the macro-crack seen in the TT photo of Fig. 4 can be understood in terms of polycrystal orientation and stress state. Figure 3 indicates that the difference in stress magnitude between the TT and IP cases presents a "softer" direction for straining orthogonal for the TT impact and the reverse situation for the IP 
impact. This softer matrix response in conjunction with preferred orientation for cleavage of the basal planes in the TT impact configuration increases void coalescence, as compared to the IP impact where "harder" orthogonal straining and misorientation of the basil planes inhibits void coalescence.
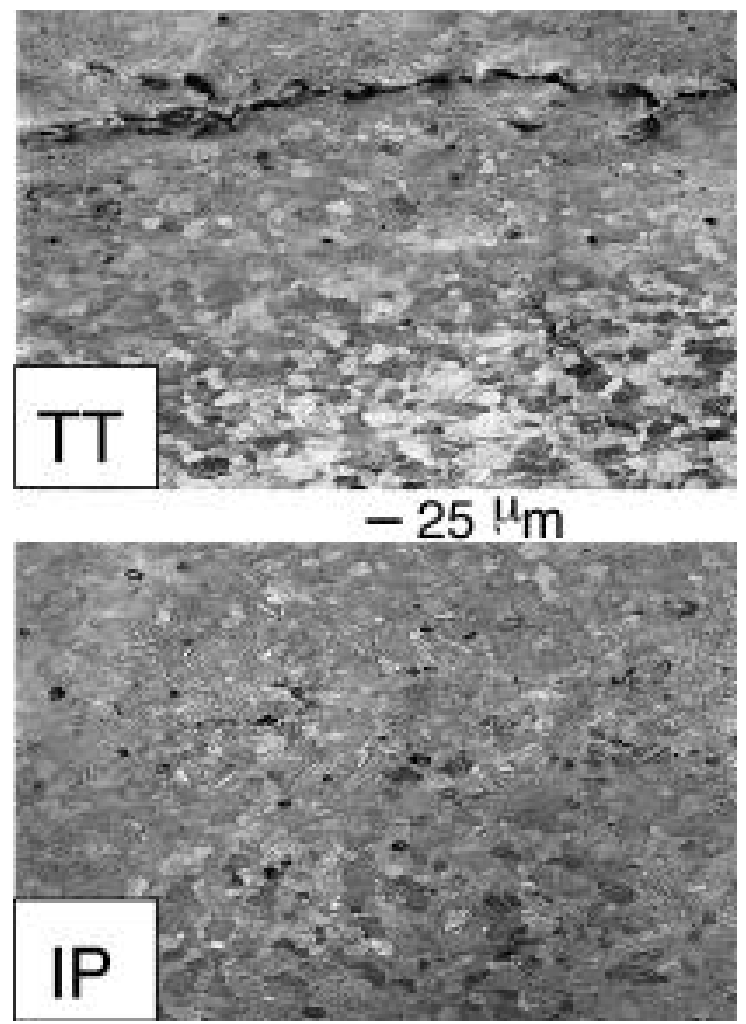

FIGURE 4. Comparison of the microstructures in recovered spalled samples for the IP and TT loading directions for $\mathrm{Zr}$

The similarities in the "pull-back" signals and damage evolution for the TT and IP samples supports the dominance of the high-strain-rate tensile-hydrostatic-stress state during spallation enforcing similar stresses to nucleate voids in $\mathrm{Zr}$ independent of crystallographic texture. This behavior is consistent with the more isotropic macroscopic response of $\mathrm{Zr}$ during high-strain-rate loading, compared to its very anisotropic quasistatic behavior, due to an increased incidence of pryramidal slip during high-strain-rate loading(2).

While no statistically quantitative void volume fraction was determined, the void fraction is estimated to be $\sim 5-10 \%$. This relatively low approximate void volume fraction is considered to be problematic given the strong magnitude of the pull-back Hopkinson-spallation signals measured for both the TT and IP samples shown in Fig. 1.

\section{SUMMARY AND CONCLUSIONS}

The effect of texture on the Hopkinson fracture "spallation" behavior of high-purity Zr was studied using VISAR, 3-D FVM simulations, and postmortem analysis of "soft" recovered spall samples. Crystallographic texture in $\mathrm{Zr}$ was found to exhibit a strong influence on the HEL magnitude, material acceleration on the free surface and damage evolution in the "soft-recovered" samples, while displaying a minimal influence on the "pull-back" signal. The influence of texture on the HEL is shown via modeling to be consistent with the orientational dependency of the elastoplastic response of $\mathrm{Zr}$.

\section{ACKNOWLEDGMENTS}

This work was supported under the auspices of the United States Department of Energy. The authors acknowledge Mike Lopez for performing the metallographic sample analysis.

\section{REFERENCES}

1. Kocks, U.F., Tomé, C.N. and Wenk, H.-R., Texture and Anisotropy, Preferred Orientations in Polycrystals and their Effect on Materials Properties, Cambridge University Press, New York, NY, 1998, pp. Pages.

2. Maudlin, P.J., Gray III, G.T., Cady, C.M. and Kaschner, G.C., Philos. Trans. Roy. Soc. 357, in press (1999).

3. Sevillano, J.G. and Meizoso, A.M., in 8th Int. Conf. on Textures of Materials (ICOTOM 8), (ed. J.S. Kallend and G. Gottstein), : The Metallurgical Society, pp. 897-911, (1988).

4. Hopkinson, B., in The Scientific Papers of Bertram Hopkinson, (ed. Cambridge : Cambridge University Press, pp. 423-437, (1921).

5. Skidmore, I.C., Appl. Mater. Res. 4, 131-147 (1965)

6. Meyers, M.A. and Aimone, C.T., Prog. Mater. Sci. 28, 1-96 (1983).

7. Pope, L.E. and Johnson, J.N., J. Appl. Phys. 46, 720729 (1975).

8. Kaschner, G.C., Gray III, G.T. and Chen, S.R., in Shock Compression of Condensed Matter - 1997, (ed. S.C. Schmidt, D.P. Dandekar, andJ.W. Forbes), Woodbury, New York : American Institute of Physics, pp. 435-438, (1998).

9. Hemsing, W.F., Rev. Sci. Instrum 50, 73-78 (1979).

10.Maudlin, P.J., Bingert, J.F., House, J.W. and Chen, S.R., Int. J. Plast. 15, 139-166 (1999).

11.O'Rourke, P.J. and Sahota, M.S., J. Comp. Phys. 143, 312-345 (1998). 
12.Chen, S.R. and Gray III, G.T., J. Phys. IV France Colloq. C3 (EURODYMAT 97) 7, 741-746 (1997). 